\title{
Relationship between biochemical indices in hemodialysis patients and mortality; survival analysis
}

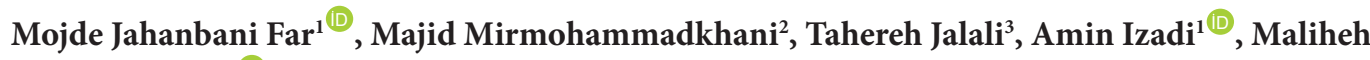 \\ Yarmohamadi $^{4^{*} \mathbb{D}}$ \\ ${ }^{1}$ Student Research Committee, Semnan University of Medical Sciences, Semnan, Iran \\ ${ }^{2}$ Social Determinants of Health Research Center, Semnan University of Medical Sciences, Semnan, Iran \\ ${ }^{3}$ Community Medicine, Tehran University of Medical Sciences, Tehran, Iran \\ ${ }^{4}$ Department of Internal Medicine, Faculty of Medicine, Semnan University of Medical Sciences, Semnan, Iran
}

\section{A R T I C L E I N F O}

Article Type:

Original

\section{Article History:}

Received: 20 August 2019

Accepted: 16 December 2019

Published online: 10 January 2020

Keywords:

Hemodialysis patients,

Premature death, Survival

analysis, Chronic kidney

disease, End-stage renal disease,

Renal replacement therapy

\begin{abstract}
A B S T R A C T
Introduction: People with chronic kidney disease (CKD) undergoing hemodialysis pay high costs and suffer from complications like premature death.

Objectives: This study was conducted to determine the relationship of various blood parameters in hemodialysis with premature death.

Patients and Methods: In this cross-sectional study, 90 hemodialysis patients were enrolled using consensus sampling method. Demographic characteristics were recorded. Additionally, serum level of ferritin and biochemical parameters were assessed. The log-rank test for survival probability and Cox proportional-hazards model were applied to examine the correlation between assessed factors and the risk of premature death.

Results: The results of log-rank test showed that only coronary artery disease (CAD) and serum ferritin have a role in predicting survival probability, since the survival rate of the people with and without CAD was about 25 months (with a confidence interval of 37.60 12.36) and 46 months (with a confidence interval of 51.63-40.36) $(P=0.041)$ respectively. Conclusion: It seems that both serum ferritin levels and cardiovascular diseases are effective factors to predict premature death in hemodialysis patients.
\end{abstract}

\section{Implication for health policy/practice/research/medical education:}

In a study on 90 hemodialysis patients we found a significant relationship between serum ferritin level and coronary artery disease with premature death in hemodialysis patients.

Please cite this paper as: Jahanbani Far M, Mirmohammadkhani M, Jalali T, Izadi A, Yarmohamadi M. Relationship between biochemical indices in hemodialysis patients and mortality; survival analysis. J Renal Inj Prev. 2020; 9(2): e16. doi: 10.15171/ jrip.2020.16.

\section{Introduction}

Chronic kidney disease (CKD) is a disorder characterized by irreversible change in structure and function of the kidney, depending on the underlying cause or causes (1). CKD has become pandemic in the world, since about 8 million adults in the United States suffer from advanced CKD (2). Hemodialysis and renal transplantation, are essential modalities for survival of patients with end-stage renal disease (ESRD) (3). Hemodialysis is conducted in more than $90 \%$ of the patients with ESRD in hospital environment $(4,5)$. Given the drastic increase in using renal replacement therapy in recent decades, its increasing trend, and the lack of kidneys available for transplant, hemodialysis is the commonest used method in these patients $(6,7)$. Global statistics show that in the last halfcentury, the extensive use of dialysis has been a great success in increasing life expectancy of ESRD patients and preventing death because of uremia (8).

In spite of beneficial therapeutic effects of hemodialysis for ESRD patients, different complications have been reported for this therapy (9). One of the most undesirable complications in dialysis patients is premature death, since 5 -year survival rate in the United States is 33\% in dialysisdiabetic patients and $50 \%$ in non-diabetic patients. Despite modern technology and new drugs, premature death has been seen in dialysis patients (10). In general, overall mortality in the first six months of dialysis is estimated to be $22 \%$ (11). This mortality rate increases during the first 
year of dialysis and reaches even to $34.4 \%$ (12).

Identifying treatable and controllable factors can prevent premature death in hemodialysis patients to a large extent. For instance, increased inflammatory factors, impaired lipid profile, red blood cell production disorder and iron metabolism and other biochemical factors have been reported as the factors associated with premature death in these patients (13). Drechsler et al showed the relationship between mortality and values of bone alkaline phosphatase (the Netherlands) (14), while Erdem et al showed the relationship between mortality and neutrophil to lymphocyte ratio (15). Additionally, Pečovnik-Balon et al (16) showed the relationship between vitamin D deficiency and mortality in these patients.

\section{Objectives}

This study was conducted to detect the relationship of premature death with related parameters in a group of hemodialysis patients.

\section{Patients and Methods \\ Study design}

The study was descriptive-analytic conducted in Semnan. Ninety patients admitted to hemodialysis ward of Kowsar hospital, (2014 to 2018), were included in the study using the consensus method. All patients were dialyzed with the same dialyzer. A checklist of demographic data (age and gender) and type of underlying disease like cardiovascular disease, hypertension and diabetes mellitus was recorded. In this study, cardiovascular diseases including myocardial infarction, coronary heart disease, angina pectoris, heart attack and ischemic heart disease were considered.

After 12 hours of overnight fasting and in the morning before starting dialysis, the blood samples were collected. We assessed the levels of hemoglobin, ferritin, cholesterol, triglyceride, serum albumin, bone alkaline phosphatase, vitamin D3 and intact parathyroid hormone (PTH). Three months after the onset of hemodialysis, up to two years, after the study patients were monitored regarding their mortality or life status. During this time, the participants were asked to observe their diet as stated by the physician and avoid arbitrary drug use without informing their physicians. Finally, the relationship between biochemical indices and the data regarding hemodialysis outcome was examined to verify the premature death or life. In this study, the phrase; "premature death" was applied for the death of an individual after three months to two years after the start of hemodialysis.

\section{Inclusion and exclusion criteria}

Inclusion criteria included signing informed consent forms and passing more than 90 days of hemodialysis. Patients with a history of chronic hemodialysis or requiring emergency dialysis, autoimmune diseases, malignancy, acute or chronic infection, a history of known liver disease, and referral to other medical centers for hemodialysis and also kidney transplantation or peritoneal dialysis were excluded.

\section{Ethical issues}

The research followed the tenets of the Declaration of Helsinki. The Ethics Committee of Semnan University of Medical Sciences approved this study (\#IR.SEMUMS. REC.1397.167). Written informed consent was taken from all participants before the study. This work has been conducted as part of residency in internal medicine thesis of Mojde Jahanbani-Far at this university (\#A-10-331-13).

\section{Statistical analysis}

The data were analyzed by IBM SPSS 23 through t-test (or Mann-Whitney U test) and chi-square test (or Fisher's exact test). The results were reported as mean \pm SD. The significant level was considered as 5\%. Moreover, log rank test was used to examine the survival probability of the patients and Cox regression model to examine the relationship between factors studied with the risk of premature death in hemodialysis patients.

Results

Patients' profile

Around 90 patients were studied, which 58.9\% (53 patients) were males. The mean age and body mass index (BMI) of patients were $63 \pm 3.5$ years and $23.6 \pm 2.4 \mathrm{~kg} /$ $\mathrm{m}^{2}$, respectively since most of the patients were in the age group of 51 to 65 years (42.2\%). There was no significant relationship between the final outcome of patients (death or life) with age, gender or BMI $(P>0.05)$. In patients, the prevalence of coronary artery disease (CAD) was $52.2 \%$, hypertension in $65.6 \%$ and diabetes mellitus presented in $64.4 \%$. The prevalence of premature death in patients suffering from CAD was significantly higher than those not suffering $(P=0.009$; Table 1$)$.

\section{Biochemistry indices}

In this study, only ferritin was significantly associated with death or life of patients $(P=0.023$; Table 2).

\section{Survival analysis}

The results of log rank test showed that only CAD and ferritin values have a role in predicting survival

Table 1. Final outcome in based on type of underlying disease

\begin{tabular}{lcccc}
\hline \multirow{2}{*}{ Underlying Disease } & \multicolumn{2}{c}{ Final Outcome } & \multirow{2}{*}{ P value } \\
\cline { 3 - 4 } & & Live & Dead & \\
\cline { 2 - 3 } & & No. (\%) & No. (\%) & \\
\hline Cardiovascular & Yes & $21(40.4)$ & $26(68.4)$ & \multirow{2}{*}{0.009} \\
diseases & No & $31(59.6)$ & $12(31.6)$ & \\
\multirow{2}{*}{ Hypertension } & Yes & $35(67.3)$ & $24(63.2)$ & \multirow{2}{*}{0.682} \\
\multirow{2}{*}{ Diabetes } & No & $17(32.7)$ & $14(36.8)$ & \\
& Yes & $31(59.6)$ & $27(71.1)$ & \multirow{2}{*}{0.263} \\
\hline
\end{tabular}


Table 2. Final outcome based on biochemical index values

\begin{tabular}{|c|c|c|c|c|}
\hline \multirow{2}{*}{ Biochemistry indices } & \multirow{2}{*}{ Total (mean \pm SD) } & \multicolumn{2}{|c|}{ Final Outcome (mean $\pm S D$ ) } & \multirow{2}{*}{$P$ value } \\
\hline & & Live & Dead & \\
\hline $\operatorname{ALP}(I U / L)$ & $349.8 \pm 222.0$ & $351.0 \pm 224.3$ & $348.1 \pm 221.7$ & 0.403 \\
\hline Albumin (g/dL) & $3.7 \pm 0.49$ & $3.78 \pm 0.50$ & $3.63 \pm 0.47$ & 0.683 \\
\hline iPTH (pg/mL) & $258.2 \pm 202.7$ & $266.6 \pm 207.9$ & $239.8 \pm 188.5$ & 0.586 \\
\hline Ferritin (ng/mL) & $387.8 \pm 375.5$ & $366.0 \pm 311.4$ & $492.3 \pm 367.7$ & $0.023 *$ \\
\hline Triglyceride (mg/dL) & $128.2 \pm 71.9$ & $134.7 \pm 81.6$ & $119.4 \pm 55.8$ & 0.822 \\
\hline Cholesterol (mg/dL) & $137.7 \pm 31.8$ & $140.6 \pm 32.4$ & $133.8 \pm 30.8$ & 0.314 \\
\hline Vitamin D3 (ng/mL) & $24.9 \pm 13.14$ & $24.71 \pm 13.52$ & $25.42 \pm 12.39$ & 0.315 \\
\hline
\end{tabular}

*Significant.

probability, since the survival rate of the people with and without CAD was about 25 months (with a confidence interval of 37.60-12.36) and 46 months (with a confidence interval of 51.63-40.36) $(P=0.041)$ respectively. Moreover, the survival rate of people with ferritin below $500 \mathrm{ng} / \mathrm{mL}$ was about 46 months since, in those with ferritin of more than $500 \mathrm{ng} / \mathrm{mL}$ was about 23 months $(P=0.43)$ (Figures 1 and 2). The results of Cox regression analysis showed the risk of premature death to be 1.89 times (approximately two times) more than those not suffering. Additionally, in the people with serum ferritin above $500 \mathrm{ng} / \mathrm{mL}$, the risk of premature death was 0.55 more than those with ferritin less than $500 \mathrm{ng} / \mathrm{mL}$.

\section{Discussion}

Our study showed, $42.2 \%$ (38 subjects) had premature death at follow-up time while $57.8 \%$ (52 subjects) survived. Yapark et al showed the overall mortality in the first 6 months of dialysis was 22\% (11). In a sevenyear study in Canada, McQuillan et al showed the 90day mortality rate for dialysis patients (in the first three months of dialysis initiation) was $34.7 \%$ (12). The reason for the low-prevalence of premature death in these studies compared to our patients seems to be the low follow-up time, because other studies have shown that this mortality rate increases with increase in the follow-up time of individuals over the first year of dialysis $(11,12)$.

There was no significant correlation between age, gender and BMI with premature death in this study. There was a significant relationship between age and premature death in the studies by Stojceva-Taneva et al in the United States (17) and Panaput et al in Thailand (13). Furthermore, McQuillan et al showed that BMI had a significant relationship with premature death (12). The results of these studies were inconsistent with our study.

Among the biochemical factors, only serum ferritin was significantly related with premature death, since the survival rates of the patients with ferritin less than $500 \mathrm{ng} /$ $\mathrm{mL}$ was about two times (46 months) than patients with serum ferritin more than $500 \mathrm{ng} / \mathrm{mL}$ (23 months). The role of ferritin in predicting premature death has been shown in studies by Karaboyas et al (18), Park et al (19), Maruyama et al (20) and Kuragano et al (21). In these studies, a significant relationship between serum ferritin levels and premature death was detected, which is in contrast with the results of our study. However, contrary to these results, in a 4-year cohort study, Kalantar-Zadeh et al, showed ferritin level had no relationship with premature death of hemodialysis patients (22). The justification for this contradiction may be due to the difference in the type of

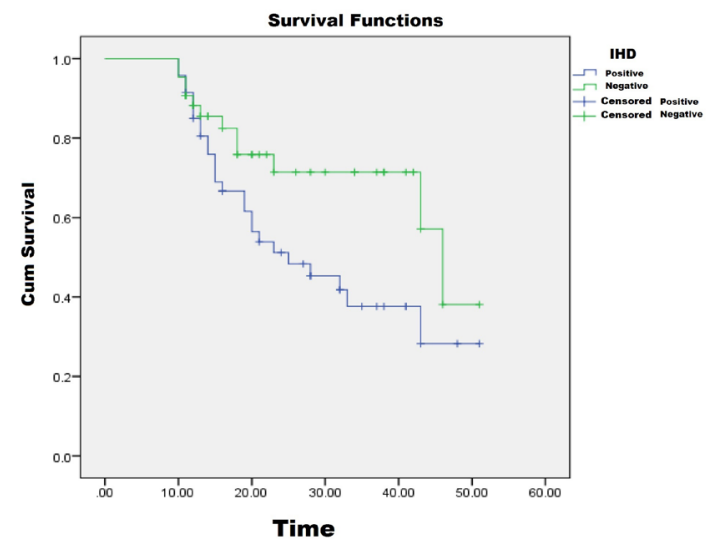

Figure 1. Survival analysis on cardiovascular disease variables.

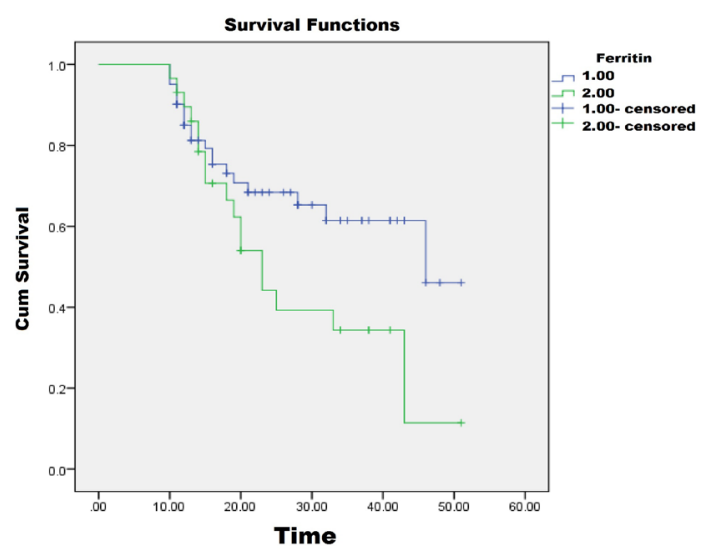

Figure 2. Survival analysis on ferritin level variables. 
iron deficiency treatment over time, the tools and method of measuring ferritin (the time of the test or kits), and the difference in the population examined in various studies. Feldman et al showed that hemodialysis patients receiving $1000 \mathrm{mg}$ or more of intravenous iron in 6-month periods had a higher probability of premature death than other patients (23). Even the prescribed dosage of iron may a role in the prevalence of premature death in hemodialysis patients (24).

The results of our study also showed that among the underlying factors, the prevalence of premature death in patients with $\mathrm{CAD}$, with a significant relationship, was higher than those not suffering the diseases. In people with CAD, the risk of premature death was 1.89 times (approximately 2 times) higher than those without the diseases. The results of some studies, in line with the results of our study, while heart failure, ischemic heart disease, cardiac arrhythmias, and severe peripheral vascular disease among the important predictors of premature death in dialysis patients $(11,12)$. Maruyama et al (20) and Kuragano et al (21) showed, higher premature death of hemodialysis patients may be due to the increased incidence of CAD. These results have been somehow effective in justifying the significant relationship between premature death of hemodialysis patients and the risk of CAD in our study.

\section{Conclusion}

There was a significant relationship between serum ferritin level and also CAD with premature death in hemodialysis patients. Examining the effects of ferritin control on decreasing premature death and survival of hemodialysis patients is suggested for further studies.

\section{Limitations of the study}

The limitations of descriptive studies, such as the inability to control all of the concomitant causes and the underlying variables are the main limitations of this study.

\section{Conflicts of interest}

There is no conflict of interest in this study.

\section{Acknowledgments}

This work has been presented as an abstract in the 17th International Congress of Nephrology, Dialysis, and Transplantation, Tabriz, Iran, 2019. We would like to thank the Clinical Research Development Unit of Kowsar Educational and Research and Therapeutic Center of Semnan University of Medical Sciences for providing facilities to this work.

\section{Authors' contribution}

MY and MJ searched the literature and prepared the primary draft. AI performed first the edition. MY conducted final edition. MMK and TJ performed statistical analysis. All authors wrote and signed the final paper.

\section{Ethical considerations}

Ethical issues (including plagiarism, data fabrication, double publication) have been completely observed by the authors.

\section{Funding/Support}

This study was supported by deputy of research of Semnan University of Medical Sciences.

\section{References}

1. Levey AS, Coresh J, Balk E, Kausz AT, Levin A, Steffes MW, et al. National Kidney Foundation practice guidelines for chronic kidney disease: evaluation, classification, and stratification. Ann Intern Med. 2003;139:137-47.

2. Kanbay M, Ikizek M, Solak Y, Selcoki Y, Uysal S, Armutcu F, et al. Uric acid and pentraxin-3 levels are independently associated with coronary artery disease risk in patients with stage 2 and 3 kidney disease. Am J Nephrol. 2011;33:32531. doi: $10.1159 / 000324916$.

3. Kara B, Açikel CH. The effect of intradialytic food intake on the urea reduction ratio and single-pool $\mathrm{Kt} / \mathrm{V}$ values in patients followed-up at a hemodialysis center. Turkish J Med Sci. 2010;40:91-97. doi: 10.3906/sag-0811-9.

4. Wen CP, Matsushita K, Coresh J, Iseki K, Islam M, Katz R, et al. Relative risks of chronic kidney disease for mortality and end-stage renal disease across races are similar. Kidney Int. 2014;86:819-27. doi: 10.1038/ki.2013.553.

5. Murphy D, McCulloch CE, Lin F, Banerjee T, BraggGresham JL, Eberhardt MS, Morgenstern H, et al. Trends in Prevalence of Chronic Kidney Disease in the United States. Ann Intern Med. 2016;165:473-81. doi: 10.7326/M16-0273.

6. Wongrakpanich S, Susantitaphong P, Isaranuwatchai S, Chenbhanich J, Eiam-Ong S, Jaber BL. Dialysis therapy and conservative management of advanced chronic kidney disease in the elderly: a systematic review. Nephron. 2017;137:178-89. doi: 10.1159/000477361.

7. Lacson E Jr, Wang W, Zebrowski B, Wingard R, Hakim RM. Outcomes associated with intradialytic oral nutritional supplements in patients undergoing maintenance hemodialysis: a quality improvement report. Am J Kidney Dis. 2012;60:591-600. doi: 10.1053/j.ajkd.2012.04.019.

8. Sameiro-Faria Md, Ribeiro S, Costa E, Mendonça D, Teixeira L, Rocha-Pereira P, et al. Risk factors for mortality in hemodialysis patients: two-year follow-up study. Dis Markers. 2013;35:791-8. doi: 10.1155/2013/518945.

9. Cole NI, Liyanage H, Suckling RJ, Swift PA, Gallagher H, Byford R, et al. An ontological approach to identifying cases of chronic kidney disease from routine primary care data: a cross-sectional study. BMC Nephrol. 2018;19:85. doi: 10.1186/s12882-018-0882-9

10. Kara E, Sahutoglu T, Ahbap E, Sakaci T, Koc Y, Basturk T, et al. The predictive value of malnutrition-inflammation score on 1-year mortality in Turkish maintenance hemodialysis patients. Clin Nephrol. 2016;86:94. doi: 10.5414/CN108799.

11. Yaprak M, Turan MN, Dayanan R, Akın S, Değirmen E, Ylldirım $M$, et al. Platelet-to-lymphocyte ratio predicts mortality better than neutrophil-to-lymphocyte ratio in 
hemodialysis patients. Int Urol Nephrol. 2016;48:1343-8. doi: $10.1007 / \mathrm{s} 11255-016-1301-4$.

12. McQuillan R, Trpeski L, Fenton S, Lok CE. Modifiable risk factors for early mortality on hemodialysis. Int J Nephrol. 2012;2012:435736. doi: 10.1155/2012/435736.

13. Panaput T, Thinkhamrop B, Domrongkitchaiporn S, Sirivongs D, Praderm L, Anukulanantachai J, et al. Dialysis dose and risk factors for death among ESRD patients treated with twice-weekly hemodialysis: a prospective cohort study. Blood Purif. 2014;38:253-62. doi: 10.1159/000368885.

14. Drechsler C, Verduijn M, Pilz S, Krediet RT, Dekker FW, Wanner C, et al. Bone alkaline phosphatase and mortality in dialysis patients. Clin J Am Soc Nephrol. 2011;6:1752-9. doi: $10.2215 / C J N .10091110$.

15. Erdem E, Coşkun K, Karataş A, Dilek M, Akpolat T. Neutrophil to lymphocyte ratio in predicting shortterm mortality in hemodialysis patients. J Exp Clin Med. 2013;30:129-32.

16. Pečovnik-Balon B, Jakopin E, Bevc S, Knehtl M, Gorenjak M. Vitamin D as a novel nontraditional risk factor for mortality in hemodialysis patients. Ther Apher Dial. 2009;13:268-72. doi: 10.1111/j.1744-9987.2009.00722.x.

17. Stojceva-Taneva O, Selim G, Tozija L, Polenakovic M. Early mortality rate in end-stage renal disease patients initiating hemodialysis. Prilozi. 2006;2:29-35.

18. Karaboyas A, Morgenstern H, Pisoni RL, Zee J, Vanholder $\mathrm{R}$, Jacobson $\mathrm{SH}$, et al. Association between serum ferritin and mortality: findings from the USA, Japan and European Dialysis Outcomes and Practice Patterns Study. Nephrol
Dial Transplant. 2018;33:2234-44. doi: 10.1093/ndt/gfy190.

19. Park KS, Ryu GW, Jhee JH, Kim HW, Park S, Lee SA, et al. Serum ferritin predicts mortality regardless of inflammatory and nutritional status in patients starting dialysis: a prospective cohort study. Blood Purif. 2015;40:209-17. doi: $10.1159 / 000438819$

20. Maruyama Y, Yokoyama K, Yokoo T, Shigematsu T, Iseki $\mathrm{K}$, Tsubakihara Y. The different association between serum ferritin and mortality in hemodialysis and peritoneal dialysis patients using Japanese nationwide dialysis registry. PloS One. 2015;10:e0143430. doi: 10.1371/journal. pone. 0143430 .

21. Kuragano T, Matsumura O, Matsuda A, Hara T, Kiyomoto $\mathrm{H}$, Murata $\mathrm{T}$, et al. Association between hemoglobin variability, serum ferritin levels, and adverse events/ mortality in maintenance hemodialysis patients. Kidney Int. 2014;86:845-54. doi: 10.1038/ki.2014.114.

22. Kalantar-Zadeh K, Regidor DL, McAllister CJ, Michael B, Warnock DG. Time-dependent associations between iron and mortality in hemodialysis patients. J Am Soc Nephrol. 2005;16:3070-80. doi: 10.1681/ASN.2005040423.

23. Feldman HI, Santanna J, Guo W, Furst H, Franklin E, Joffe $\mathrm{M}$, et al. Iron administration and clinical outcomes in hemodialysis patients. J Am Soc Nephrol. 2002;13:734-44.

24. Feldman HI, Joffe M, Robinson B, Knauss J, Cizman B, Guo W, et al. Administration of parenteral iron and mortality among hemodialysis patients. J Am Soc Nephrol. 2004;15:1623-32. doi: 10.1097/01.asn.0000128009.69594.be

Copyright ( 2020 The Author(s); Published by Nickan Research Institute. This is an open-access article distributed under the terms of the Creative Commons Attribution License (http://creativecommons.org/licenses/by/4.0), which permits unrestricted use, distribution, and reproduction in any medium, provided the original work is properly cited. 